\title{
A systematic review of non-invasive brain stimulation therapies and cardiovascular risk: implications for the treatment of major depressive disorder
}

\section{Leonardo Augusto Negreiros Parente Capela Sampaio ${ }^{1}$, Renerio Fraguas ${ }^{1}$, Paulo Andrade Lotufo ${ }^{2,3}$, Isabela Martins Benseñor ${ }^{2,3}$ and André Russowsky Brunoni ${ }^{2,4}$ *}

1 Department of Psychiatry, University of São Paulo Medical School, São Paulo, Brazil

${ }^{2}$ Clinical Research Center, University Hospital, University of São Paulo, São Paulo, Brazil

${ }^{3}$ Department of Internal Medicine, University of São Paulo Medical School, São Paulo, Brazil

${ }^{4}$ Department of Neurosciences and Behavior, Institute of Psychology, University of São Paulo, São Paulo, Brazil

\section{Edited by:}

Zafiris J. Daskalakis, University of Toronto, Canada

\section{Reviewed by:}

Paul Croarkin, Mayo Clinic, USA

Daniel Blumberger, Centre for

Addiction and Mental Health, Canada

${ }^{*}$ Correspondence:

André Russowsky Brunoni, Centro de Pesquisas Clínicas, Hospital

Universitário, Av. Prof Lineu Prestes 2565, 30 andar, Sao Paulo, Sao Paulo, Brazil.

e-mail: brunoni@usp.br
Major depressive disorder (MDD) and cardiovascular diseases are intimately associated. Depression is an independent risk factor for mortality in cardiovascular samples. Neuroendocrine dysfunctions in MDD are related to an overactive hypothalamus-pituitary-adrenal (HPA) axis and increased sympathetic activity. Novel intervention strategies for MDD include the non-invasive brain stimulation (NIBS) techniques such as repetitive transcranial magnetic stimulation (rTMS) and transcranial direct current stimulation (tDCS). In fact, although these techniques have being increasingly used as a treatment for MDD, their cardiovascular effects were not sufficiently investigated, which would be important considering the dyad MDD/cardiovascular disorders. We investigated this issue through a systematic review for published articles from the first date available to May 2012 in MEDLINE and other databases, looking for main risk factors and surrogate markers for cardiovascular disease such as: cortisol, heart rate variability (HRV), alcohol, smoking, obesity, hypertension, glucose. We identified 37 articles (981 subjects) according to our eligibility criteria. Our main findings were that NIBS techniques might be effective strategies for down-regulating HPA activity and regulating food, alcohol, and cigarette consumption. NIBS's effects on HRV and blood pressure presented mixed findings, with studies suggesting that HRV values can decrease or remain unchanged after NIBS, while one study found that rTMS increased blood pressure levels. Also, a single study showed that glucose levels decrease after tDCS. However, most studies tested the acute effects after one single session of rTMS/tDCS; therefore further studies are necessary to investigate whether NIBS modifies cardiovascular risk factors in the long-term. In fact, considering the burden of cardiac disease, further trials in cardiovascular, depressed, and non-depressed samples using NIBS should be performed.

Keywords: transcranial direct current stimulation, major depressive disorder, repetitive transcranial magnetic stimulation, systematic review, heart rate variability, hypothalamo-hypophyseal system

\section{INTRODUCTION}

Major depressive disorder (MDD) and cardiovascular disorders are both prevalent and disabling conditions. It is expected that, by 2020 , they are going to be the second and the first main causes of disability, respectively, worldwide (Murray and Lopez, 1997). These disorders present a complex relationship, with one increasing the prevalence and severity of the other - e.g., subjects with depression have an increased risk for myocardial infarction (MI) while those with depression post-MI have increased risk of mortality (Taylor et al., 2005; Whooley et al., 2008). However, pharmacological treatments do not necessarily diminish cardiovascular risk, as shown in recent clinical trials in patients with cardiovascular disease and depression (Jiang et al., 2011). Further, tricyclic antidepressants and antiepileptic drugs (also used in some types of depression) decrease heart rate variability (HRV), which is a surrogate marker of cardiovascular illness (Kemp et al., 2010; Lotufo et al., 2012). Likewise, tricyclics might also increase levels of IL-6 and TNF-alpha that are pro-inflammatory cytokines associated with cardiovascular risk (Maes et al., 2011). Therefore, the development of treatments that are not only clinically effective but also safe from a cardiovascular perspective is needed.

In recent years, non-invasive brain stimulation (NIBS) techniques, such as repetitive transcranial magnetic stimulation (rTMS) and transcranial direct current stimulation (tDCS), have been investigated as putative treatments for depression. Transcranial magnetic stimulation (TMS) depolarizes neurons through a potent, relatively focal, electromagnetic field that is generated beneath a coil positioned over the scalp. When applied repetitively, rTMS has therapeutic effects. Recent meta-analyses demonstrated 
that high-frequency (HF) rTMS (i.e., excitatory) over the left dorsolateral prefrontal cortex (DLPFC) and low-frequency (LF) rTMS (i.e., inhibitory) over the right DLPFC are effective treatments for MDD (Schutter, 2009, 2010). In contrast to TMS, tDCS applies weak, direct electric currents to the brain through relatively large electrodes placed over the scalp (Brunoni et al., 2012a). For MDD, the anode is placed over the area corresponding to the left DLPFC. Recently, a meta-analysis (Kalu et al., 2012) and a large randomized, clinical trial (Brunoni et al., in press) suggested that tDCS might be an effective treatment for depression.

Therefore, considering that (1) cardiovascular disease and depression are related conditions; (2) antidepressants might have a hazardous impact in increasing cardiovascular risk and (3) NIBS therapies are potential therapeutic interventions for depression, we sought to systematically review the effects of such techniques on cardiovascular risk factors. We looked for risk factors such as increased blood pressure and serum glucose levels, obesity, alcohol use, and smoking. We also included in our search strategy HRV and hypercortisolism that are markers of cardiovascular risk. Importantly, we did not look specifically for studies with MDD, as we anticipated that the total number of studies would be low. Rather, our aim was to summarize current evidence of the effects of NIBS techniques on cardiovascular risk factors and discuss their implications in the treatment of MDD. Nevertheless, our findings can be generalized for other neuropsychiatric disorders in which pharmacological treatment is associated with hazardous cardiovascular effects.

\section{MATERIALS AND METHODS}

We performed a systematic review for published articles from the first date available to May 2012 in the following databases: MEDLINE, Scielo, and Web of Knowledge. According to our proposal, the key search terms were:

\#1 “TMS” (MeSH term) OR “tDCS” OR (“transcranial” AND "stimulation")

\#2 "Hypothalamo-hypophyseal system" (MeSH term) OR "hypothalamus-pituitary-adrenal (HPA) axis" OR "cortisol"

\#3 "HRV" OR "RR variability"

\#4 "Smoking"

\#5 "Obesity" (MeSH term) OR "food”

\#6 [“Alcohol” AND (“dependence" OR "abuse”)] OR "alcohol”

\#7 "Blood pressure" OR "hypertension"

\#8 "Glucose" OR “diabetes"

The key search term \#1 was crossed with (Boolean term AND) each other term. We included all original clinical studies written in English, apart from studies in experimental animals. We also examined reference lists in retrieved papers, and contacted experts on the field for identifying additional articles.

\section{RESULTS}

Figure 1 depicts the search strategy and included articles. Table 1 describes each article individually. We identified 37 articles, corresponding to 40 studies, since some articles reported more than one cardiovascular risk factor. We further discuss NIBS effects on each risk factor.

\section{HPA AXIS}

Masur et al. (1991) evaluated cortisol plasma levels in healthy volunteers, observing no specific rTMS effects. Later, Evers et al. (2001) evaluated healthy subjects in a sham-controlled design, finding that rTMS decreased cortisol serum levels; whereas Baeken et al. (2009b) performed a sham-controlled study in healthy women, finding no rTMS effects on the HPA axis. However, Baeken et al. (2011) found, in another sample, that rTMS decreased cortisol levels when controlling for baseline state anxiety. Other studies reported that rTMS decreased salivary cortisol levels in patients with depression (Baeken et al., 2009a) and bulimia (Claudino et al., 2010). Zwanzger et al. (2003, 2007) evaluated rTMS effects on the "area under the curve" of cortisol response in two studies: in depressed patients, the authors explored whether therapeutical rTMS would change the cortisol response to the dexamethasone cortisol suppression test that is characteristically impaired in depression, finding no rTMS effects. Later on, the authors evaluated whether rTMS would decrease panic symptomatology and cortisol response after experimentally induction of a panic attack with CCK-4. They also found no specific rTMS effects.

Raimundo et al. (2012) evaluated tDCS effects on cortisol plasma levels in healthy volunteers, with no specific effects. Conversely, Binkofski et al. (2011) observed that anodal tDCS decreased cortisol serum levels compared to sham. Finally, Brunoni et al. (2012b) also found cortisol levels decreasing after anodal tDCS coupled with negative imagery viewing, which is a method to experimentally induce stress.

\section{HEART RATE VARIABILITY}

Yoshida et al. (2001) suggested that rTMS could activate the sympathetic nervous system as LF power increased - however, LF reflects both sympathetic and parasympathetic activity (ESC, 1996) and therefore a more appropriate conclusion would be that rTMS activated both the sympathetic and parasympathetic systems. Udupa et al. (2007), in a randomized, controlled trial compared HRV changes after escitalopram and rTMS in depressed subjects, suggesting that rTMS not only improved depression but also restored sympathovagal balance (i.e., increased HRV values), in contrast with escitalopram. Finally, Goldie et al. (2010) described that rTMS led to "decreased arousal levels" (i.e., decreased sympathetic/increased parasympathetic activity), although the study primary aim was to identify novel HRV measures, and not rTMS effects on HRV per se.

Regarding tDCS, Vandermeeren et al. (2010) applied anodal, cathodal, and sham tDCS in 30 healthy volunteers, finding no specific effects on HRV. Conversely, Montenegro et al. (2011) applied anodal tDCS over the left temporal area, observing HF HRV increasing and LF-HRV decreasing; however, this effect was observed only in highly fit athletes. Finally, Brunoni et al. (2012b) found that anodal tDCS increased HF-HRV, particularly during negative imagery viewing, suggesting that tDCS could interfere favorably in stress responses elicited by negative imagery.

\section{BLOOD PRESSURE}

In a safety study, Jahanshahi et al. (1997) evaluated HF rTMS in six healthy subjects, finding a trend $(p=0.06)$ for active 


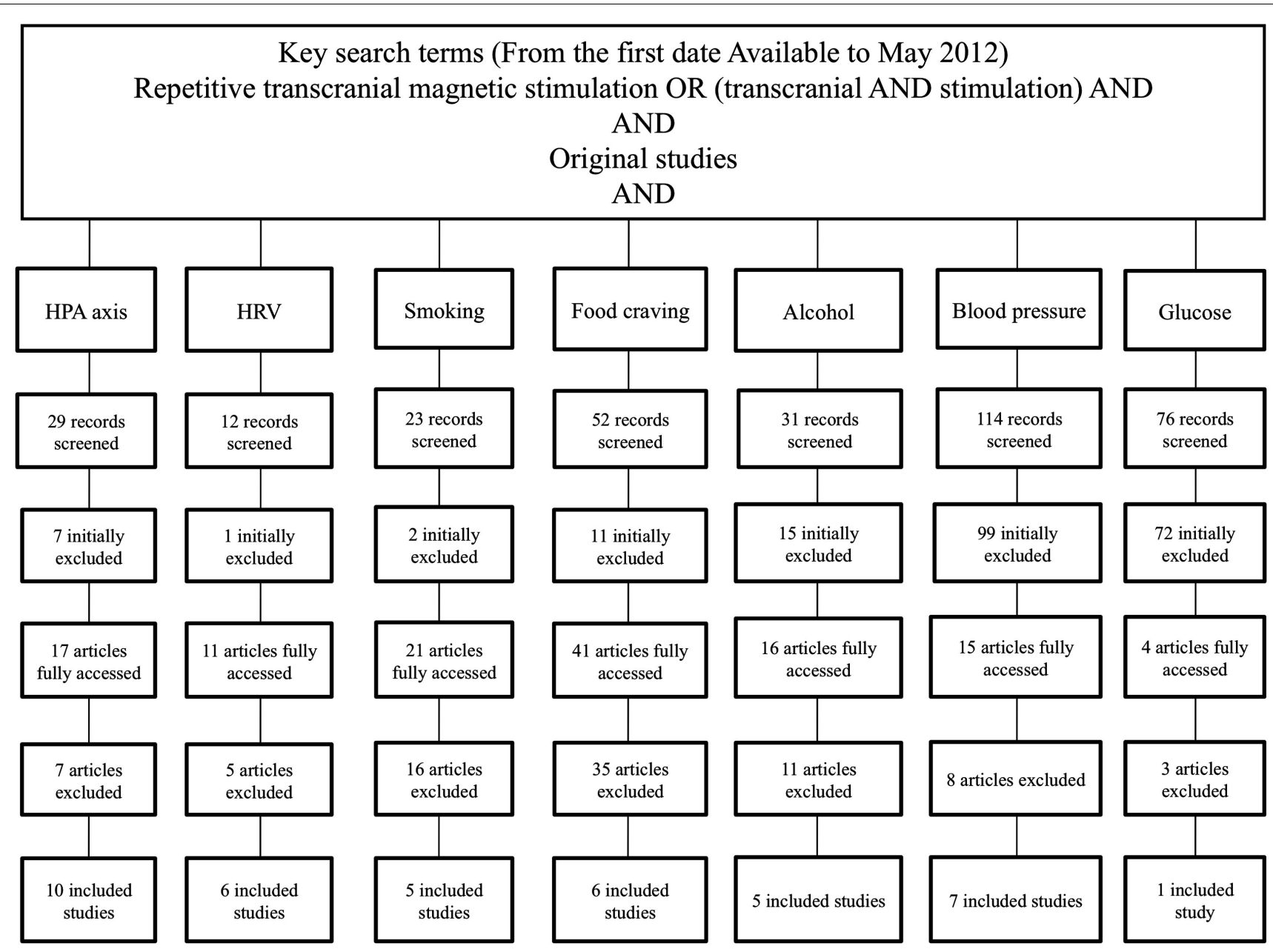

FIGURE 1 | Flow chart of reviewed studies, according to PRISMA guidelines. The complete key search terms are described in the main text.

rTMS decreasing blood pressure. Jenkins et al. (2002) compared the effects of rTMS on mood and blood pressure over the left and right DLPFC finding that left but not right stimulation decreased arterial pressure. Likewise, Knotkova et al. (2012), in an open-label study, found that anodal tDCS over the left DLPFC generally decreased blood pressure levels. In addition, Binkofski et al. (2011) used anodal tDCS over the left primary motor cortex, finding small but significant decrements on blood pressure.

Conversely, Yozbatiran et al. (2009) found a small but significant increase of systolic blood pressure after HF rTMS stimulation over the ipsilesional primary motor cortex hand area in stroke patients. Other studies with rTMS (Van den Eynde et al., 2010) and tDCS (Vandermeeren et al., 2010) showed no changes in blood pressure.

\section{SERUM GLUCOSE LEVELS}

Here, no rTMS studies matching our eligibility criteria were found. For tDCS, Binkofski et al. (2011) studied male healthy volunteers after active and sham tDCS stimulation. They observed increased systemic glucose uptake tolerance in the active group.

\section{FOOD INTAKE}

Fregni et al. (2008b) found that desire for food consumption and visual attention for food imagery decreased after tDCS, in a shamcontrolled design with healthy volunteers. Similar findings were demonstrated in subsequent tDCS (Goldman et al., 2011) and rTMS (Camus et al., 2009; Van den Eynde et al., 2010) studies. Uher et al. (2005) found that self-reported food craving during exposure to food remained stable before and after active rTMS, while it increased after sham stimulation. However, Barth et al. (2011) found that rTMS inhibited food craving no better than sham rTMS.

\section{SMOKING}

Fregni et al. (2008a) compared active vs. sham tDCS over the left DLPFC in smokers and found specific tDCS effects in decreasing cue-induced craving. In fact, another study showed that such effects were cumulative over 5 days (Boggio et al., 2009). Amiaz et al. (2009) reported similar findings, and also that rTMS decreased cigarette consumption. Conversely, Eichhammer et al. (2003) reported that rTMS decreased cigarette smoking although not levels of craving. Finally, Rose et al. (2011) found that 
Table 1 | Summary of reviewed studies.

\begin{tabular}{|c|c|c|c|c|}
\hline & $\boldsymbol{N}$ & Sample & Protocol & Findings \\
\hline \multicolumn{5}{|c|}{ HYPOTHALAMIC-PITUITARY-ADRENAL (HPA) SYSTEM } \\
\hline Masur et al. (1991) & 10 & HS & HF-rTMS at F3 & rTMS had no impact on cortisol levels \\
\hline Evers et al. (2001) & 23 & HS & HF-rTMS at F3 & rTMS mildly decreased cortisol levels \\
\hline Zwanzger et al. (2003) & 37 & MDD & HF-rTMS at F3 & rTMS did not affect cortisol levels after the DEX/CRH test \\
\hline Zwanzger et al. (2007) & 11 & HS & LF-rTMS at F4 & rTMS did not affect cortisol levels after panic induction with CCK-4 \\
\hline Baeken et al. (2009a) & 20 & MDD & HF-rTMS at F3 & rTMS decreased cortisol immediately and after $30 \mathrm{~min}$ \\
\hline Baeken et al. (2009b) & 54 & HS & HF-rTMS at F3 or F4 & rTMS had no impact on salivary cortisol levels \\
\hline Baeken et al. (2011) & 24 & HS & HF-rTMS at F4 & Baseline anxiety modified cortisol expression after rTMS \\
\hline Raimundo et al. (2012) & 50 & HS & atDCS at C3 & rTMS had no impact on cortisol levels \\
\hline Binkofski et al. (2011) & 15 & HS & atDCS at M3 & atDCS decreased cortisol levels \\
\hline Brunoni et al. (2012b) & 20 & HS & $\begin{array}{l}\text { atDCS at F3/ctDCS at F4 and } \\
\text { vice-versa }\end{array}$ & atDCS at F3 decreased cortisol levels \\
\hline \multicolumn{5}{|c|}{ HEART RATE VARIABILITY (HRV) } \\
\hline Yoshida et al. (2001) & 32 & $\mathrm{HS}$ & LF-rTMS at Cz & rTMS increased low-frequency HRV \\
\hline Udupa et al. (2007) & 52 & MDD & HF-rTMS at F3 & rTMS improved sympathovagal balance \\
\hline Goldie et al. (2010) & 6 & HS & Unclear & HRV effectively measures arousal levels \\
\hline Vandermeeren et al. (2010) & 30 & HS & atDCS or ctDCS at Fz & No differences in HRV between groups \\
\hline Montenegro et al. (2011) & 20 & HS & atDCS at T3 & Sympathovagal balance decreased only in athletes \\
\hline Brunoni et al. (2012b) & 20 & HS & $\begin{array}{l}\text { aTDCS at F3/ctDCS at F4 and } \\
\text { vice-versa }\end{array}$ & atDCS at F3 increased high-frequency HRV during negative imagery \\
\hline \multicolumn{5}{|l|}{ BLOOD PRESSURE (BP) } \\
\hline Jahanshahi et al. (1997) & 6 & HS & HF-rTMS at M3 & HF-rTMS showed a trend to decrease BP $(p=0.06)$ \\
\hline Jenkins et al. (2002) & 19 & HS & LF-rTMS at F3 or F4 & Left but not right rTMS decreased BP \\
\hline Yozbatiran et al. (2009) & 12 & Stroke & HF-rTMS at M1 & rTMS increased BP \\
\hline Vandermeeren et al. (2010) & 30 & HS & atDCS at $\mathrm{Fz}$ & No differences in BP between groups \\
\hline Van den Eynde et al. (2011) & 38 & BN & HF-rTMS at F3 & No effects in BP between groups \\
\hline Binkofski et al. (2011) & 15 & $\mathrm{HS}$ & atDCS at M3 & tDCS decreased BP \\
\hline Knotkova et al. (2012) & 8 & MDD & aTDCS at F3 & BP initially increased and then decreased after tDCS \\
\hline \multicolumn{5}{|l|}{ GLUCOSE LEVELS } \\
\hline Binkofski et al. (2011) & 15 & HS & atDCS at M3 & tDCS increased glucose tolerance \\
\hline \multicolumn{5}{|l|}{ FOOD INTAKE } \\
\hline Uher et al. (2005) & 28 & HS & HF-rTMS at F3 & Food craving increased after sham, but not after real stimulation \\
\hline Fregni et al. (2008b) & 23 & HS & $\begin{array}{l}\text { aTDCS at F3/ctDCS at F4 and } \\
\text { vice-versa }\end{array}$ & tDCS decreased food craving \\
\hline Camus et al. (2009) & 56 & HS & HF-rTMS at F4 & rTMS decreased increased evaluation for food \\
\hline Van den Eynde et al. (2010) & 38 & BN & HF-rTMS at F3 & rTMS decreased urge to eat and binge-eating episodes \\
\hline Barth et al. (2011) & 10 & HS & HF-rTMS at F3 & Prefrontal rTMS inhibited food cravings no better than sham rTMS \\
\hline Goldman et al. (2011) & 19 & HS & atDCS at F4/ctDCS at F3 & tDCS decreased food craving \\
\hline \multicolumn{5}{|l|}{ NICOTINE DEPENDENCE } \\
\hline Eichhammer et al. (2003) & 14 & Smokers & HF-rTMS at F3 & rTMS reduced cigarette smoking \\
\hline Fregni et al. (2008a) & 24 & Smokers & atDCS at F3 or F4 & Left or right tDCS reduced craving \\
\hline Amiaz et al. (2009) & 48 & Smokers & HF-rTMS at F3 & rTMS reduced cigarette consumption and craving \\
\hline Boggio et al. (2009) & 27 & Smokers & atDCS at F3 & tDCS decreased craving after smoking cue exposure \\
\hline Rose et al. (2011) & 15 & Smokers & HF-rTMS and LF-rTMS at SFG & Differential effects of craving according to type of stimulation \\
\hline \multicolumn{5}{|c|}{ ALCOHOL DEPENDENCE (AD) } \\
\hline Boggio et al. (2008) & 13 & $A D$ & aTDCS at F3/ctDCS at F4 & tDCS decreased alcohol craving \\
\hline Mishra et al. (2010) & 45 & $A D$ & HF-rTMS at F4 & rTMS had significant anticraving effects in alcohol dependence \\
\hline Höppner et al. (2011) & 19 & $A D$ & HF-rTMS at F3 & No differences in craving and mood between groups \\
\hline Herremans et al. (2012) & 36 & $A D$ & HF-rTMS at F4 & No significant effects on alcohol craving \\
\hline Nakamura-Palacios et al. (2012) & 49 & $A D$ & HF-rTMS at F3 & tDCS increased frontal activity for Lesch IV alcoholics \\
\hline
\end{tabular}

HS, healthy subjects; MDD, major depressive disorder; $B N$, bulimia nervosa; $A D$, alcohol dependence; HF-and LF-rTMS, high-frequency and low-frequency repetitive transcranial magnetic stimulation, respectively; atDCS, anodal transcranial direct current stimulation; ctDCS, cathodal transcranial direct current stimulation; BP, blood

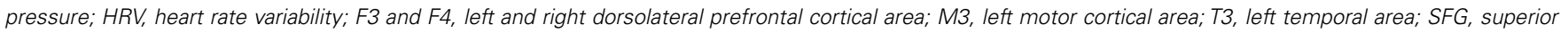
frontal gyrus. 
HF-rTMS over the superior frontal gyrus increased craving after smoking cue presentations, while it decreased craving after neutral cue ones.

\section{ALCOHOL CONSUMPTION}

Herremans et al. (2012) showed no rTMS effects on alcohol craving in alcohol dependent patients. Conversely, Mishra et al. (2010) reported positive findings for rTMS in reducing alcohol craving. Höppner et al. (2011) showed mixed findings: although rTMS had no effect on alcohol craving, the authors found that real vs. sham rTMS increased the attentional blink on alcohol-related pictures.

Regarding tDCS, Boggio et al. (2008) found that active tDCS decreased alcohol craving in patients with alcohol dependence. Further, Nakamura-Palacios et al. (2012) found that active vs. sham tDCS increased cognitive performance and prefrontal activity in Lesch IV alcoholics.

\section{DISCUSSION}

In this systematic review, we evaluated 37 studies (981 subjects) addressing the effects of NIBS techniques on several risk factors. We found that: (1) cortisol levels decrease or remain unchanged after NIBS; (2) NIBS effects on HRV and blood pressure presented mixed findings, with studies suggesting that HRV values can decrease or remain unchanged after NIBS (one study found that rTMS increased blood pressure levels); (3) a single study showed that glucose levels decrease after tDCS; (4) most studies found that NIBS can effectively decrease craving for alcohol, smoking, and food intake. We further discuss these findings.

First, some limitations should be underscored. Although the studies generally presented adequate methodology in terms of design and assessment, most of them explored the effects of NIBS after one single session. In addition, most studies were exploratory, without a clearly defined hypothesis. Therefore, the evidence here presented should be considered preliminary and hypothesis-driven for further studies.

The effects of NIBS on the HPA axis and cardiovascular mechanisms are likely to occur via a top-down modulation, i.e., changes in cortical activity that subsequently modify the activity of centers related to hormonal and cardiovascular regulation that are situated in subcortical areas and in the brain stem. Of note, NIBS effects on these systems occurred mostly in the context of stress response e.g., negative image visualization (Brunoni et al., 2012b) and $16 \mathrm{~h}$ fasting (Binkofski et al., 2011) or in clinical samples, such as depression (Baeken et al., 2009a), bulimia (Claudino et al., 2010), and anxiety (Baeken et al., 2011). Likewise, such effects occurred primarily during neuromodulation of DLPFC, an area implicated in the pathophysiology of stress and affective disorders (Koenigs and Grafman, 2009). This suggests that NIBS effects might not act on these systems during their steady state; but rather when they are being activated - for instance, during a stress response. Further studies should investigate in which conditions blood pressure and cortisol expression can be modulated by NIBS; and also which brain areas should be targeted. In this context, using computer simulated models and/or neuroimaging studies to identify which brain areas are activated during brain stimulation might help to identify cortical areas that could be targeted in order to indirectly modulate the subcortical and brain stem areas related to cardiovascular control. Another option is to investigate the use of novel NIBS techniques, such as "deep rTMS" (Levkovitz et al., 2007) and "high-definition" tDCS (Minhas et al., 2010) that can theoretically target deeper brain areas and/or induce more focalized stimuli as to directly modulate those areas.

Most reviewed studies showed that NIBS effectively regulated craving and consumption for food, alcohol, and cigarette smoking. This is in agreement with the putative role of the prefrontal cortex as a brain region related to decision-making, either of non-emotional ("cold cognition") and emotional ("hot cognition") content (Koenigs and Grafman, 2009), the latter including also the inhibitory control of appetitive impulses. This brain area is also connected with subcortical areas related to the "reward system," which includes the ventral tegmental area and nucleus accumbens (Fecteau et al., 2010). Modulation of food, alcohol, and cigarette intake could be a crucial contribution of NIBS for the cardiovascular field, since most risk factors are directly associated with poor dietary and lifestyle choices. However, since most studies evaluated the acute effects of tDCS, further investigation should focus on the long-term effects of tDCS in regulating craving.

Finally, Binkofski et al. (2011) observed increased systemic glucose turnover with active tDCS. This is a proxy for insulin resistance that is impaired in diabetes mellitus. The authors suggested that their findings could be explained by the activation of ATP-dependent potassium channels (KATP) by tDCS in the hypothalamus, which would inhibit hepatic gluconeogenesis. Further studies could evaluate whether tDCS modifies other mechanisms of insulin metabolism in healthy and clinical samples.

\section{IMPLICATIONS FOR MDD TREATMENT}

The activation of the HPA axis is an important link between MDD and cardiovascular illness (Maes et al., 2011). Hypercortisolism leads to a dysregulation of glucose, lipid metabolism, and blood pressure, which contributes to the metabolic syndrome. Moreover, hypercortisolism has unfavorable effects on the immune system. In our review, we observed that some NIBS studies decreased cortisol levels, suggesting that these techniques might induce modulatory top-down effects that lead to a down-regulation of the HPA axis. Further research should explore whether such effects persist over time - for instance, evaluating other cortisol measures, such as cortisol awakening response and cortisol daily curve.

Major depressive disorder is also related to an autonomic shift toward sympathetic predominance, which also increases inflammatory response in the endothelium and platelet activation, hastening the formation of atherosclerotic plaques (Lett et al., 2004). This sympathetic tone shift boosts sympatho-adrenal response, increasing catecholamines secretion in adrenal medulla, leading to a hypercortisolemic and hypercatecholaminergic state, contributing to this physiopathological mechanism. Our results showed that HRV is either non-affected or increased after NIBS (Evers et al., 2001). A possible mechanism of action for NIBS is also topdown modulation, through activity increasing in cortical regions and down-regulation of the sympatho-adrenomedullary system, leading to a decrease in sympathetic activity. 


\section{OTHER BRAIN STIMULATION TECHNIOUES}

Although NIBS seems to down-regulate stress response, this might not necessarily be true to other forms of brain stimulation. For instance, electroconvulsive therapy (ECT) has a characteristic biphasic response, i.e., a parasympathetic phase that lasts for approximately $20 \mathrm{~s}$ followed by a sympathetic phase of approximately $1 \mathrm{~min}$ (Geersing et al., 2011). In addition, repetitive trans-spinal magnetic stimulation, i.e., a TMS coil applied over the sixth and seventh cervical vertebrae, showed no difference between sham and active groups on measures of HRV (Paxton et al., 2011). However, deep brain stimulation seems to have favorable effects over the autonomic nervous system in patients with Parkinson's disease, in contrast to levodopa that has unfavorable effects (Ludwig et al., 2007).

Another potentially interesting therapy is vagus nerve stimulation (VNS). Recently, a HRV meta-analysis of epilepsy and epilepsy treatment suggested that could have favorable effects on

\section{REFERENCES}

Amiaz, R., Levy, D., Vainiger, D., Grunhaus, L., and Zangen, A. (2009). Repeated high-frequency transcranial magnetic stimulation over the dorsolateral prefrontal cortex reduces cigarette craving and consumption. Addiction 104, 653-660.

Baeken, C., De Raedt, R., Leyman, L., Schiettecatte, J., Kaufman, L., Poppe, K., et al. (2009a). The impact of one HF-rTMS session on mood and salivary cortisol in treatment resistant unipolar melancholic depressed patients. J. Affect. Disord. 113, 100-108.

Baeken, C., De Raedt, R., Leyman, L., Schiettecatte, J., Poppe, K., Kaufman, L., et al. (2009b). The impact of one session of HF-rTMS on salivary cortisol in healthy female subjects. World J. Biol. Psychiatry 10, 586-590.

Baeken, C., Vanderhasselt, M. A., and De Raedt, R. (2011). Baseline 'state anxiety' influences HPA-axis sensitivity to one sham-controlled HFrTMS session applied to the right dorsolateral prefrontal cortex. Psychoneuroendocrinology 36, 60-67.

Bajbouj, M., Merkl, A., Schlaepfer, T. E., Frick, C., Zobel, A., Maier, W., et al. (2010). Two-year outcome of vagus nerve stimulation in treatment-resistant depression. J. Clin. Psychopharmacol. 30, 273-281.

Barth, K. S., Rydin-Gray, S., Kose, S., Borckardt, J. J., O'neil, P. M., Shaw, D., et al. (2011). Food cravings and the effects of left prefrontal repetitive transcranial magnetic stimulation using an improved sham condition. Front. Psychiatry 2:9. doi:10.3389/fpsyt.2011.00009

Binkofski, F., Loebig, M., Jauch-Chara, K., Bergmann, S., Melchert, U. H., Scholand-Engler, H. G., et al. (2011).
Brain energy consumption induced by electrical stimulation promotes systemic glucose uptake. Biol. Psychiatry 70, 690-695.

Boggio, P. S., Liguori, P., Sultani, N., Rezende, L., Fecteau, S., and Fregni, F. (2009). Cumulative priming effects of cortical stimulation on smoking cue-induced craving. Neurosci. Lett. 463, 82-86.

Boggio, P. S., Sultani, N., Fecteau, S., Merabet, L., Mecca, T., PascualLeone, A., et al. (2008). Prefrontal cortex modulation using transcranial DC stimulation reduces alcohol craving: a double-blind, sham-controlled study. Drug Alcohol Depend. 92, 55-60.

Brunoni, A. R., Valiengo, L., Baccaro, A., Zanao, T. A., Oliveira, A. C., Goulart, A. C., et al. (in press). The sertraline versus electrical current therapy for treating depression clinical trial (SELECT TDCS): results from a factorial, randomized, controlled trial. Arch. Gen. Psychiatry.

Brunoni, A. R., Nitsche, M. A., Bolognini, N., Bikson, M., Wagner, T., Merabet, L., et al. (2012a). Clinical research with transcranial direct current stimulation (tDCS): challenges and future directions. Brain Stimul. 5, 175-195.

Brunoni, A. R., Vanderhasselt, M. A., Boggio, P. S., Fregni, F., Dantas, E. M., Mill, J. G., et al. (2012b). Polarity- and valencedependent effects of prefrontal transcranial direct current stimulation on heart rate variability and salivary cortisol. Psychoneuroendocrinology. PMID: 22626867. [Epub ahead of print]

Camus, M., Halelamien, N., Plassmann, H., Shimojo, S., O'doherty, J., Camerer, C., et al. (2009). Repetitive

sympathovagal balance, presumably through direct stimulation of the vagus nerve (Lotufo et al., 2012). Further, it also seems to be a safe therapy from a cardiovascular perspective, according to a recent long-term follow-up in depressed patients (Bajbouj et al., 2010).

\section{CONCLUSION}

We found that NIBS techniques, used in neuropsychology and neuropsychiatry, might also exert effects in pathological endocrine, immune, and neural events associated with cardiovascular disease, particularly in managing the cardiovascular reactivity induced by stress and inhibiting food and cigarette consumption. For MDD, these techniques contrast with antidepressants not only due to the absence of pharmacological interaction, but also possibly in terms of cardiovascular effects. Nevertheless, further studies are needed to determine the scope of these interventions, defining their effect power and indications for regulating cardiovascular and metabolic parameters.

transcranial magnetic stimulation over the right dorsolateral prefrontal cortex decreases valuations during food choices. Eur. J. Neurosci. 30, 1980-1988.

Claudino, A. M., Van Den Eynde, F., Stahl, D., Dew, T., Andiappan, M. Kalthoff, J., et al. (2010). Repetitive transcranial magnetic stimulation reduces cortisol concentrations in bulimic disorders. Psychol. Med. PMID: 20925970. [Epub ahead of print].

Eichhammer, P., Johann, M., Kharraz, A., Binder, H., Pittrow, D., Wodarz, N., et al. (2003). Highfrequency repetitive transcranial magnetic stimulation decreases cigarette smoking. J. Clin. Psychiatry 64, 951-953.

ESC. (1996). Heart rate variability. Standards of measurement, physiological interpretation, and clinical use. Task Force of the European Society of Cardiology and the North American Society of Pacing and Electrophysiology. Eur. Heart J. 17, 354-381.

Evers, S., Hengst, K., and Pecuch, P. W. (2001). The impact of repetitive transcranial magnetic stimulation on pituitary hormone levels and cortisol in healthy subjects. J. Affect. Disord. 66, 83-88.

Fecteau, S., Fregni, F., Boggio, P. S. Camprodon, J. A., and PascualLeone, A. (2010). Neuromodulation of decision-making in the addictive brain. Subst. Use. Misuse 45 1766-1786.

Fregni, F., Liguori, P., Fecteau, S., Nitsche, M. A., Pascual-Leone, A., and Boggio, P. S. (2008a). Cortical stimulation of the prefrontal cortex with transcranial direct current stimulation reduces cue-provoked smoking craving: a randomized, sham-controlled study. J. Clin. Psychiatry 69, 32-40.

Fregni, F., Orsati, F., Pedrosa, W., Fecteau, S., Tome, F. A., Nitsche, M. A., et al. (2008b). Transcranial direct current stimulation of the prefrontal cortex modulates the desire for specific foods. Appetite 51, 34-41.

Geersing, P. G., Bulte, C. S., Viersen, V. A., Stek, M. L., Bouwman, R. A., Boer, C., et al. (2011). Beat-tobeat hemodynamic monitoring during electroconvulsive therapy. J. ECT 27, 189-191.

Goldie, J., McGregor, C., and Murphy, B. (2010). Determining levels of arousal using electrocardiography: a study of HRV during transcranial magnetic stimulation. Conf. Proc. IEEE Eng. Med. Biol. Soc. 2010 , 1198-1201.

Goldman, R. L., Borckardt, J. J., Frohman, H. A., O'neil, P. M., Madan, A., Campbell, L. K., et al. (2011). Prefrontal cortex transcranial direct current stimulation (tDCS) temporarily reduces food cravings and increases the selfreported ability to resist food in adults with frequent food craving. Appetite 56, 741-746.

Herremans, S. C., Baeken, C., Vanderbruggen, N., Vanderhasselt, M. A. Zeeuws, D., Santermans, L., et al. (2012). No influence of one rightsided prefrontal HF-rTMS session on alcohol craving in recently detoxified alcohol-dependent patients: results of a naturalistic study. Drug Alcohol Depend. 120, 209-213.

Höppner, J., Broese, T., Wendler, L., Berger, C., and Thome, J. (2011). Repetitive transcranial magnetic stimulation (rTMS) for treatment of alcohol dependence. World J. Biol. Psychiatry 12(Suppl. 1), 57-62. 
Jahanshahi, M., Ridding, M. C., Limousin, P., Profice, P., Fogel, W., Dressler, D., et al. (1997). Rapid rate transcranial magnetic stimulation-a safety study. Electroencephalogr. Clin. Neurophysiol. 105, 422-429.

Jenkins, J., Shajahan, P. M., Lappin, J. M., and Ebmeier, K. P. (2002). Right and left prefrontal transcranial magnetic stimulation at $1 \mathrm{~Hz}$ does not affect mood in healthy volunteers. BMC Psychiatry 2, 1. doi:10.1186/1471244X-2-1

Jiang, W., Krishnan, R., Kuchibhatla, M., Cuffe, M. S., Martsberger, C., Arias, R. M., et al. (2011). Characteristics of depression remission and its relation with cardiovascular outcome among patients with chronic heart failure (from the SADHARTCHF Study). Am. J. Cardiol. 107, 545-551.

Kalu, U. G., Sexton, C. E., Loo, C. K., and Ebmeier, K. P. (2012). Transcranial direct current stimulation in the treatment of major depression: a meta-analysis. Psychol. Med. 42, 1791-1800.

Kemp, A. H., Quintana, D. S., Gray, M. A., Felmingham, K. L., Brown, K., and Gatt, J. (2010). Impact of depression and antidepressant treatment on heart rate variability: a review and meta-analysis. Biol. Psychiatry 67, 1067-1074.

Knotkova, H., Rosedale, M., Strauss, S. M., Horne, J., Soto, E., Cruciani, R. A., et al. (2012). Using transcranial direct current stimulation to treat depression in HIV-infected persons: the outcomes of a feasibility study. Front. Psychiatry 3:59. doi:10.3389/fpsyt.2012.00059

Koenigs, M., and Grafman, J. (2009). The functional neuroanatomy of depression: distinct roles for ventromedial and dorsolateral prefrontal cortex. Behav. Brain Res. 201, 239-243.

Lett, H. S., Blumenthal, J. A., Babyak, M. A., Sherwood, A., Strauman, T., Robins, C., et al. (2004). Depression as a risk factor for coronary artery disease: evidence, mechanisms, and treatment. Psychosom. Med. 66, 305-315.

Levkovitz, Y., Roth, Y., Harel, E. V., Braw, Y., Sheer, A., and Zangen, A. (2007). A randomized controlled feasibility and safety study of deep transcranial magnetic stimulation. Clin. Neurophysiol. 118, 2730-2744.

Lotufo, P. A., Valiengo, L., Bensenor, I. M., and Brunoni, A. R. (2012). A systematic review and meta-analysis of heart rate variability in epilepsy and antiepileptic drugs. Epilepsia 53, 272-282.
Ludwig, J., Remien, P., Guballa, C., Binder, A., Binder, S., Schattschneider, J., et al. (2007). Effects of subthalamic nucleus stimulation and levodopa on the autonomic nervous system in Parkinson's disease. J. Neurol. Neurosurg. Psychiatr. 78, 742-745.

Maes, M., Ruckoanich, P., Chang, Y. S., Mahanonda, N., and Berk, M. (2011). Multiple aberrations in shared inflammatory and oxidative \& nitrosative stress (IO\&NS) pathways explain the co-association of depression and cardiovascular disorder (CVD), and the increased risk for CVD and due mortality in depressed patients. Prog. Neuropsychopharmacol. Biol. Psychiatry 35, 769-783.

Masur, H., Ludolph, A. C., Hilker, E., Hengst, K., Knuth, U., Rolf, L. H., et al. (1991). Transcranial magnetic stimulation: influence on plasma levels of hormones of the anterior pituitary gland and of cortisol? Funct. Neurol. 6, 59-63.

Minhas, P., Bansal, V., Patel, J., Ho, J. S., Diaz, J., Datta, A., et al. (2010). Electrodes for high-definition transcutaneous DC stimulation for applications in drug delivery and electrotherapy, including tDCS. J. Neurosci. Methods 190, 188-197.

Mishra, B. R., Nizamie, S. H., Das, B., and Praharaj, S. K. (2010). Efficacy of repetitive transcranial magnetic stimulation in alcohol dependence: a sham-controlled study. Addiction 105, 49-55.

Montenegro, R. A., Farinatti Pde, T., Fontes, E. B., Soares, P. P., Cunha, F. A., Gurgel, J. L., et al. (2011). Transcranial direct current stimulation influences the cardiac autonomic nervous control. Neurosci. Lett. 497, 32-36.

Murray, C. J., and Lopez, A. D. (1997). Alternative projections of mortality and disability by cause 19902020: global burden of disease study. Lancet 349, 1498-1504.

Nakamura-Palacios, E. M., De Almeida Benevides, M. C., Da Penha ZagoGomes, M., De Oliveira, R. W., De Vasconcellos, V. F., De Castro, L. N., et al. (2012). Auditory eventrelated potentials (P3) and cognitive changes induced by frontal direct current stimulation in alcoholics according to Lesch alcoholism typology. Int. J. Neuropsychopharmacol. 15, 601-616.

Paxton, R. J., Malcolm, M. P., Newsom, S. A., Richards, J. C., Rynn, G. M., and Bell, C. (2011). Sympathetic responses to repetitive trans-spinal magnetic stimulation. Clin. Auton. Res. 21, 81-87.
Raimundo, R. J., Uribe, C. E., and BrasilNeto, J. P. (2012). Lack of clinically detectable acute changes on autonomic or thermoregulatory functions in healthy subjects after transcranial direct current stimulation (tDCS). Brain Stimul. 5, 196-200.

Rose, J. E., McClernon, F. J., Froeliger, B., Behm, F. M., Preud'homme, X., and Krystal, A. D. (2011). Repetitive transcranial magnetic stimulation of the superior frontal gyrus modulates craving for cigarettes. Biol. Psychiatry 70, 794-799.

Schutter, D. J. (2009). Antidepressant efficacy of high-frequency transcranial magnetic stimulation over the left dorsolateral prefrontal cortex in double-blind sham-controlled designs: a meta-analysis. Psychol. Med. 39, 65-75.

Schutter, D. J. (2010). Quantitative review of the efficacy of slowfrequency magnetic brain stimulation in major depressive disorder. Psychol. Med. 40, 1789-1795.

Taylor, C. B., Youngblood, M. E., Catellier, D., Veith, R. C., Carney, R. M., Burg, M. M., et al. (2005). Effects of antidepressant medication on morbidity and mortality in depressed patients after myocardial infarction. Arch. Gen. Psychiatry 62, 792-798.

Udupa, K., Sathyaprabha, T. N. Thirthalli, J., Kishore, K. R., Raju, T. R., and Gangadhar, B. N. (2007). Modulation of cardiac autonomic functions in patients with major depression treated with repetitive transcranial magnetic stimulation. J. Affect. Disord. 104, 231-236.

Uher, R., Yoganathan, D., Mogg, A. Eranti, S. V., Treasure, J., Campbell, I. C., et al. (2005). Effect of left prefrontal repetitive transcranial magnetic stimulation on food craving. Biol. Psychiatry 58, 840-842.

Van den Eynde, F., Claudino, A.M., Campbell, I., Horrell, L., Andiappan, M., Stahl, D., et al. (2011). Cardiac safety of repetitive transcranial magnetic stimulation in bulimic eating disorders. Brain Stimul. 4, 112-114.

Van den Eynde, F., Claudino, A. M. Mogg, A., Horrell, L., Stahl, D., Ribeiro, W., et al. (2010). Repetitive transcranial magnetic stimulation reduces cue-induced food craving in bulimic disorders. Biol. Psychiatry 67, 793-795.

Vandermeeren, Y., Jamart, J., and Ossemann, M. (2010). Effect of tDCS with an extracephalic reference electrode on cardio-respiratory and autonomic functions. $\mathrm{BMC} \mathrm{Neu-}$ rosci. 11,38. doi:10.1186/1471-220211-38

Whooley, M. A., De Jonge, P., Vittinghoff, E., Otte, C., Moos, R., Carney, R.
M., et al. (2008). Depressive symptoms, health behaviors, and risk of cardiovascular events in patients with coronary heart disease. JAMA 300, 2379-2388.

Yoshida, T., Yoshino, A., Kobayashi, Y., Inoue, M., Kamakura, K., and Nomura, S. (2001). Effects of slow repetitive transcranial magnetic stimulation on heart rate variability according to power spectrum analysis. J. Neurol. Sci. 184, 77-80.

Yozbatiran, N., Alonso-Alonso, M., See, J., Demirtas-Tatlidede, A., Luu, D., Motiwala, R. R., et al. (2009). Safety and behavioral effects of high-frequency repetitive transcranial magnetic stimulation in stroke. Stroke 40, 309-312.

Zwanzger, P., Baghai, T. C., Padberg, F., Ella, R., Minov, C., Mikhaiel, P., et al. (2003). The combined dexamethasone-CRH test before and after repetitive transcranial magnetic stimulation (rTMS) in major depression. Psychoneuroendocrinology 28, 376-385.

Zwanzger, P., Eser, D., Volkel, N., Baghai, T. C., Moller, H. J., Rupprecht, R., et al. (2007). Effects of repetitive transcranial magnetic stimulation (rTMS) on panic attacks induced by cholecystokinin-tetrapeptide (CCK4). Int. J. Neuropsychopharmacol. 10, 285-289.

Conflict of Interest Statement: The authors declare that the research was conducted in the absence of any commercial or financial relationships that could be construed as a potential conflict of interest.

Received: 04 July 2012; accepted: 20 September 2012; published online: 10 October 2012.

Citation: Sampaio LANPC, Fraguas R, Lotufo PA, Benseñor IM and Brunoni $A R$ (2012) A systematic review of non-invasive brain stimulation therapies and cardiovascular risk: implications for the treatment of major depressive disorder. Front. Psychiatry 3:87. doi: 10.3389/fpsyt.2012.00087

This article was submitted to Frontiers in Neuropsychiatric Imaging and Stimulation, a specialty of Frontiers in Psychiatry. Copyright (c) 2012 Sampaio, Fraguas, Lotufo, Benseñor and Brunoni. This is an open-access article distributed under the terms of the Creative Commons Attribution License, which permits use, distribution and reproduction in other forums, provided the original authors and source are credited and subject to any copyright notices concerning any third-party graphics etc. 